\title{
EUGENIA DYSENTERICA DC: ACTIVIDAD ANTIOXIDANTE, PERFIL DE ÁCIDOS GRASOS Y DETERMINACIÓN DE TOCOFEROLES
}

\section{EUGENIA DYSENTERICA DC: ANTIOXIDANT ACTIVITY, FATTY ACIDS PROFILE AND TOCOPHEROLS DETERMINATION}

\author{
Neuza Jorge (1), Débora Maria Moreno L. (1), Bruna Jorge Bertanha (2) \\ (1) Departamento de Engenharia e Tecnologia de Alimentos, Instituto de Biociências, Letras e Ciências Exatas, \\ Universidade Estadual Paulista, São José do Rio Preto, Brasil. \\ (2) Departamento de Engenharia de Alimentos, Faculdade de Zootecnia e Engenharia de Alimentos, \\ Universidade de São Paulo, Pirassununga, Brasil.
}

\begin{abstract}
This work aimed to characterize the proximate composition, antioxidant activity and fatty acids profile and tocopherol composition of the oil from Eugenia dysenterica DC seeds. To obtain the extract, the dehydrated and triturated seeds were extracted with ethyl alcohol for 30 minutes, at a proportion of 1:3 of seeds:ethyl alcohol, under continuous agitation at room temperature. Afterwards, the mixture was filtered and the supernatant was dehydrated at $40^{\circ} \mathrm{C}$ in order to determine by direct weighing the dry matter yield of the extract. The "cagaita" seeds showed to be an important source of total carbohydrates, had relevant antioxidant activity and a high content of total phenolic compounds. In comparison to other oils and fats, "cagaita" seeds oil do not constitutes a good source of tocopherols.
\end{abstract}

Key words: seeds; antioxidant activity; phenolic compounds; DPPH :

Este trabajo fue recibido el 4 de Diciembre de 2009 y aceptado para ser publicado el 1 de Marzo de 2010.

\section{INTRODUCCIÓN}

Eugenia dysenterica DC (Myrtaceae) es un árbol fructífero nativo de los "cerrados" de hasta $10 \mathrm{~m}$ de altura, de tronco y ramas tortuosas, cáscara gruesa, fisurada (1). Su distribución es bastante amplia, siendo más común en los estados de Goiás, Minas Gerais y Bahia, en "cerrados" y "cerradões". Aparece con alta frecuencia en algunas regiones, formando grandes agrupamientos.

El fruto, popularmente conocido como "cagaita", tiene forma de globo, color amarillo claro, ácido, epicarpo membranoso, con peso entre 14 y $20 \mathrm{~g}$, longitud de 3 a $4 \mathrm{~cm}$ y diámetro de 3 a $5 \mathrm{~cm}(2,3)$.

Los frutos de "cagaita" son consumidos in natura o procesados para la obtención de licores, helados, jugos y jaleas. De su pulpa se puede obtener vinagre y alcohol (4). Esta especie forma parte de la flora apícola del "cerrado" brasileño y, sus hojas y cáscaras son utilizadas en la medicina popular como antidiarreico, para diabetes e ictericia. Tiene potencial como planta ornamental, y proveedora de corteza, además, su madera puede ser empleada para obras de construcción civil, leña y carbón (5).

Sus semillas, de coloración crema y formato oval, achatado o elipsoide, miden de 0,8 a 2,0 cm de diámetro. Presentan superficie lisa y generalmente poseen dos cotiledones. Un kilogramo de semillas contiene cerca de 700 a 1600 unidades (6).

En algunos estudios sobre la composición nutricional de diversas frutas nativas del "cerrado" brasileño se verificó que la "cagaita" posee un elevado contenido de agua $(95,01 \%)$, siendo una de las frutas que presentan mayor porcentaje de ácidos grasos poliinsaturados (linoleico e linolénico), después de la almendra de baru y de la pulpa da mangaba. Tiene mayor contenido de ácido linoleico (10,5\%) que el aceite de oliva y de dendê. En cuanto al contenido de ácido linolénico $(11,86 \%)$, supera el del aceite de maiz, girasol, cacauhete, soja, oliva y dendê. Los ácidos grasos juegan un papel muy importante en el organismo humano, siendo el linoleico 
y el linolénico los más esenciales. Son precursores de algunas sustancias en la estructura de membranas celulares, como componentes cerebrales, de la retina y del sistema reproductor (7).

Los contenidos de vitamina C de la "cagaita" $(18,28$ $\mathrm{mg} / 100 \mathrm{~g}$ ) son superiores a los encontrados en muchas frutas convencionalmente cultivadas, como la banana madura y la manzana Argentina, de 6,4 e 5,9 mg/100 g, respectivamente (8).

Innumerables compuestos naturales encontrados en frutas, cereales y vegetales presentan actividad antioxidante. Entre los más importantes antioxidantes naturales están los compuestos fenólicos (flavonoides, ácidos fenólicos y taninos), compuestos nitrogenados (alcaloides, aminoácidos, peptideos, aminas y derivados de la clorofila), carotenoides, tocoferoles y ácido ascórbico (9). Los compuestos fenólicos son antioxidantes primarios que actúan como terminales para los radicales libres (10).

Estudios clínicos y epidemiológicos han mostrado evidencias de que los antioxidantes fenólicos de cereales, frutas y vegetales son los principales factores que contribuyen en la disminución de la incidencia de enfermedades crónicas y degenerativas encontradas en poblaciones, cuyas dietas son altas en el consumo de estos alimentos (11). De esta forma, la importancia de investigar en antioxidantes naturales ha aumentado significativamente en los últimos años.

Las semillas de frutas no han recibido mucha atención como fuente de antioxidantes naturales, debido a la falta de popularidad y de aplicación industrial de las mismas. Tales circunstancias explican la importancia de la utilización eficiente de semillas, una vez que el aprovechamiento de la especie fructífera Eugenia dysenterica DC puede constituir una actividad económica bastante promisoria, dada la excelente calidad de sus frutos y sus diversas utilidades.

El presente estudio tuvo por objetivo principal caracterizar las semillas de Eugenia dysenterica DC en cuanto a su composición proximal, determinar el potencial antioxidante del aceite extraído, evaluar su perfil de ácidos grasos y su composición de tocoferoles.

\section{MATERIALES Y MÉTODOS Muestras}

Los frutos de Eugenia dysenterica DC fueron recolectados de árboles ubicados en la Universidade Estadual Paulista, São José do Rio Preto-SP, en su mayor grado de madurez correspondiente a la época de verano del año 2008. Las semillas fueron retiradas manualmente de los frutos y lavadas ligeramente con agua destilada para remover residuos de pulpas y azúcares solubles y después fueron seleccionadas.

Las semillas fueron colocadas en una estufa a $35^{\circ} \mathrm{C}$ durante 48 horas, para reducir su contenido de humedad. Después, las semillas fueron homogeneizadas para análisis posteriores por triplicado.

\section{Obtención del extracto de las semillas}

Para la obtención del extracto de Eugenia dysenterica DC, las semillas fueron trituradas y extraídas con alcohol etílico por 30 minutos, en la proporción de 1:3 de semillas:alcohol etílico, bajo agitación continua, a temperatura ambiente. Luego, la mezcla fue filtrada y el sobrenadante deshidratado en rotavapor bajo presión reducida a $40^{\circ} \mathrm{C}$ para determinar, por pesaje directo, el rendimiento en materia seca del extracto.

\section{Determinación de la composición proximal}

Las determinaciones analíticas de humedad, materia grasa y cenizas en las semillas fueron realizadas de acuerdo con los métodos estándares AOCS (12). Las proteínas fueron determinadas mediante el método Kjeldahl descrito por la AOAC (13) y los carbohidratos totales fueron cuantificados por diferencia del valor obtenido de la sumatoria de humedad, materia grasa, proteínas y cenizas.

\section{Medida de la capacidad de secuestrar radicales libres (DPPH')}

Este procedimiento fue descrito por Brand-Williams et al. (14). Se preparó una solución etanólica de $500 \mu \mathrm{g} / \mathrm{mL}$ de concentración de extracto de semillas de Eugenia dysenterica DC (solución madre), a partir de la cual se obtuvieron soluciones de 5, 10, 25, 50, 125 y $250 \mathrm{mg} / \mathrm{mL}$. Una alícuota de cada una de estas soluciones $(0,3 \mathrm{~mL})$ fue adicionada $2,7 \mathrm{~mL}$ de 1,1 -difenil-2picrilhidrazil (DPPH·), Sigma marca, solución (40 mg/ $\mathrm{mL}$ ). Después del tiempo de reacción de 30 minutos, la absorbancia fue leída a $515 \mathrm{~nm}$ y convertida en porcentaje de actividad antioxidante (AA) por medio de la siguiente ecuación:

$\mathrm{AA}(\%)=100-\left\{\left[\left(\mathrm{Abs}_{\text {muestra }}-\mathrm{Abs}_{\text {blanco }}\right) \times 100\right] / \mathrm{Abs}_{\text {control }}\right\}$

Un control fue hecho con 2,7 mL de $\mathrm{DPPH}^{\circ}$ y un blanco fue realizado con $0,3 \mathrm{~mL}$ de solución etanólica del extracto y $2,7 \mathrm{~mL}$ de etanol, para cada concentración.

\section{Compuestos fenólicos totales}

La cuantificación de los compuestos fenólicos totales fue determinada por espectrofotometría, por medio 
del reactivo de Folin-Ciocalteu, según la metodología descrita por Singleton y Rossi (15).

En este procedimiento, se pipeteó $100 \mu \mathrm{L}$ de la solución de extracto natural en tubos de ensayo y se agregó $500 \mu \mathrm{L}$ del reactivo de Folin-Ciocalteau. Luego, se agregó 1,5 mL de solución saturada de carbonato de sodio al $20 \%$ y $6 \mathrm{~mL}$ de agua destilada.

Esta mezcla se mantuvo en reposo por 2 horas a temperatura ambiente, y la absorbancia fue determinada a $765 \mathrm{~nm}$. Para realizar la curva estándar se utilizó ácido gálico y el resultado fue expresado en miligramos de equivalentes de ácido gálico por gramo de extracto $(\mathrm{mg} / \mathrm{g})$.

\section{Perfil de ácidos grasos por cromatografía gaseosa}

Los ésteres metílicos de los ácidos grasos presentes en los aceites fueron obtenidos mediante el método descrito por Hartman y Lago (16).

Para el análisis cromatográfico de ácidos grasos se utilizó un cromatógrafo de gases marca Varian (Walnut Creek, USA), modelo GC 3900, equipado con detector de ionización de llama, inyector y muestreador automático. Los compuestos fueron separados en una columna capilar de sílica fundida CP-Sil 88 de $50 \mathrm{~m}$ de largo, con diámetro interno de $0,25 \mathrm{~mm}$ y espesor de película de $0,20 \mathrm{~mm}$.

La programación de temperatura de la columna fue iniciada a $50^{\circ} \mathrm{C}$ por 2 minutos, seguida por un calentamiento con una elevación de la temperatura de $4^{\circ} \mathrm{C} /$ min hasta $240^{\circ} \mathrm{C}$ y mantenida en isoterma durante 20,5 minutos. Las temperaturas utilizadas en el inyector y en el detector fueron 230 y $250^{\circ} \mathrm{C}$, respectivamente. Las muestras inyectadas fueron de $1 \mathrm{~mL}$ de volumen, adoptándose la relación de 1:30. El gas portador fue el hidrógeno con un caudal de $30 \mathrm{~mL} / \mathrm{min}$.

Los ácidos grasos fueron identificados por comparación con los tiempos de retención de los estándares puros de ésteres metílicos de ácidos grasos con los tiempos correspondientes de los componentes separados de las muestras y la cuantificación fue hecha por normalización del área (\%). Se utilizó como estándar una mezcla compuesta de 37 ésteres metílicos de ácidos grasos (Supelco, Bellefonte, USA), de C4:0 a C24:1, con pureza entre 99,1 y $99,9 \%$.

\section{Tocoferoles}

El contenido de tocoferoles, expresados en ppm, presentes en el aceite extraído de las semillas, se determinó mediante HPLC de acuerdo al método oficial AOCS Ce 8-86 (12). Se utilizó un cromatógrafo HPLC Varian (Walnut Creek, USA), modelo 210, con detector de fluorescencia con longitud de onda de excitación a $290 \mathrm{~nm}$ y de emisión de $330 \mathrm{~nm}$. La columna utilizada fue Varian Microsorb 100 - 5 Si 250 x 4,6 mm, tamaño de partícula alrededor de $5 \mu \mathrm{m}$. La fase móvil utilizada fue hexano:isopropanol 99,5:0,5, con un caudal de 1,2 $\mathrm{mL} / \mathrm{min}$.

Análisis estadístico. Los resultados de la composición proximal, perfil de ácidos grasos y tocoferoles fueron expresados como mediana de 3 determinaciones.

\section{RESULTADOS}

En la tabla 1 se presentan las medias \pm desviación estándar de la composición proximal de las semillas de Eugenia dysenterica DC.

Según los resultados, las semillas frescas de Eugenia dysenterica DC tenían un contenido de humedad del 57,60\%. Después de la deshidratación en una incubadora a $35^{\circ} \mathrm{C}$ durante 48 horas para obtener el extracto, el contenido de humedad de las semillas fue de $32,15 \%$.

La tabla 2 presenta el rendimiento porcentual, el porcentaje máximo de actividad antioxidante $\left(\mathrm{AA}_{\text {máxima }}\right)$, el valor de $\mathrm{EC}_{50}(\mu \mathrm{g} / \mathrm{mL})$ y el contenido de compuestos fenólicos totales $(\mathrm{mg} / \mathrm{g})$ del extracto de semillas de

\section{TABLA 1}

Composición proximal de las semillas (porcentaje).

Componentes

Humedad

Materia grasa

Proteínas

Cenizas

Carbohidratos totales
Media

$32,15 \pm 0,28$

$2,36 \pm 0,31$

$4,91 \pm 0,16$

$1,18 \pm 0,05$

59,40 
Eugenia dysenterica DC.

El rendimiento en extracto seco fue $3,75 \%$ en función del solvente utilizado. Según datos publicados por Fernandes et al. (17), éste porcentaje puede variar en función de la especie del fruto y de la técnica usada para la extracción.
La tabla 3 presenta los ácidos grasos identificados por la cromatografía de gases, presentes en la materia grasa de las semillas de Eugenia dysenterica DC. A composición en ácidos grasos de las semillas muestra un contenido de $37,66 \%$ de ácidos grasos saturados con longitud de cadena entre 10 y 24 átomos de carbono,

\section{TABLA 2}

Determinaciones de rendimiento, actividad antioxidante ( $\left.\mathrm{AA}_{\text {máxima }}\right)$ y compuestos fenólicos totales del extracto de las semillas.

\section{Determinaciones}

Rendimiento (\%)

AAmáxima (\%)

$\operatorname{EC50}(\mu \mathrm{g} / \mathrm{mL})$

Compuestos fenólicos totales $(\mathrm{mg} / \mathrm{g})^{*}$

\section{Extracto de semillas}

\section{3,75}

95,92

40,63

136,66

$\mathrm{EC}_{50}$ es definido como la concentración suficiente para obtener 50\% del efecto máximo, estimado en $100 \%$.

* mg de equivalentes de ácido gálico por g de extracto.

\section{TABLA 3}

Perfil de ácidos grasos del aceite de las semillas (porcentaje).

\section{Ácidos grasos*}

Cáprico (C 10:0)

Palmítico (C 16:0)

Palmitoleico (C 16:1)

Esteárico (C 18:0)

Oleico (C 18:1 n9)

Linoleico (C 18:2 n6)

Araquidico (C 20:0)

a-linolénico (C 18:3 n3)

Henecosanoico (C 21:0)

Behénico (C 22:0)

Lignocérico (C 24:0)

Saturados

Monoinsaturados

Poliinsaturados

Sat/Insat**

Ole/Lin***

\section{Média}

$3,12 \pm 0,19$

$25,12 \pm 0,91$

$1,01 \pm 0,07$

$1,80 \pm 0,21$

$20,17 \pm 1,23$

$38,11 \pm 0,57$

$1,82 \pm 0,08$

$3,05 \pm 0,09$

$0,38 \pm 0,31$

$2,82 \pm 0,16$

$2,60 \pm 0,16$

$37,66 \pm 0,54$

$21,18 \pm 1,16$

$41,15 \pm 0,65$

$1 / 1,66$

$1 / 1,89$

*Ésteres metílicos de los ácidos grasos.

**Relación entre el total de ácidos grasos saturados e insaturados.

***Relación entre el total de ácidos oleico y linoleico. 
entre los cuales el ácido palmítico con un promedio de $25,12 \%$ es el mayoritario.

En la tabla 4 se resume la composición de tocoferoles presentes en el aceite de las semillas. El aceite de semillas contiene los isómeros $\alpha-, \beta-, \gamma-, \delta$-tocoferol, con un valor promedio total de $28,7 \mathrm{mg} / \mathrm{kg}$.

\section{DISCUSIÓN}

Composición proximal. De acuerdo con los resultados obtenidos, las semillas presentaron $32,15 \%$ de humedad y elevado contenido de carbohidratos totales $(59,40 \%)$. Se observaron bajos porcentajes de materia grasa $(2,36 \%)$ y proteínas $(4,91 \%)$.

Actividad antioxidante. La actividad antioxidante de los compuestos, dada por el valor de EC50, se calcula a partir de una reducción del $50 \%$ de la concentración inicial de $\mathrm{DPPH}^{\circ}$. Cabe destacar que a menor valor de $\mathrm{EC}_{50}$, mayor es la actividad antioxidante del compuesto analizado. El valor de $\mathrm{EC}_{50}$, obtenido por regresión lineal, para el extracto de semillas, mostró un elevado coeficiente de regresión, que fue $\mathrm{R}^{2}=0,9030$. Los valores de actividad antioxidante máxima y $\mathrm{EC}_{50}$ alcanzados por el extracto de semillas fueron de $95,92 \%$ y $40,63 \mathrm{mg} /$ $\mathrm{mL}$, respectivamente.

Compuestos fenólicos. La concentración de compuestos fenólicos totales encontrada fue de 136,66 mg de equivalentes de ácido gálico por gramo de extracto de semillas. La extracción de compuestos fenólicos de productos naturales es fuertemente influenciada por el solvente utilizado. Se ha observado que cuanto mayor es la polaridad del solvente de extracción, mayor es la cantidad de compuestos fenólicos extraídos (18).

Ácidos grasos. Los resultados indican que el extracto etanólico tiene un grande potencial antioxidante y estudios adicionales serán necesarios para evaluar esa propriedad y su aplicación en los alimentos.

La cantidad total de ácidos grasos insaturados fue de $62,33 \%$, de los cuales $21,18 \%$ son ácidos monoinsaturados y 41,15\% ácidos poliinsaturados, siendo el ácido linoleico su principal componente. El ácido $\alpha$-linolénico se encuentra en cantidad de 3,05\%. Entre los ácidos monoinsaturados y poliinsaturados, sólo el ácido oleico $(20,17 \%)$ y el ácido linoleico $(38,11 \%)$ se encuentran en cantidades significativas.

La calidad y digestibilidad de los aceites vegetales comestibles son determinadas por la cantidad y composición de ácidos grasos insaturados. La presencia de ácido linoleico en contenidos adecuados es fundamental, ya que se trata de un ácido graso esencial. Cuanto mayor es la cantidad de ácido linoleico en relación al oleico, mejor es la calidad del aceite vegetal para evitar la formación del colesterol LDL (19).

En el aceite de palma, Corsini et al. (20) encontraron una relación entre ácidos grasos saturados e insaturados de $1 / 1,06$, indicando que este aceite puede ser utilizado en frituras, en cuanto la estabilidad oxidativa está relacionada a la cantidad de ácidos grasos insaturados.

De acuerdo con la tabla 3, el aceite de semillas de Eugenia dysenterica DC presentó una relación ácido oleico/linoleico (Ole/Lin) de 1/1,89, similar al valor encontrado por Borges et al. (21) para el aceite de semillas de umbu $(1 / 1,05)$.

Tocoferoles. Como puede observarse, contiene $95 \%$ de $\alpha$-tocoferol con un valor promedio total de $27,2 \mathrm{mg} /$ $\mathrm{kg}$, lo que significa, que en comparación con otros aceites y grasas, el aceite de estas semillas no constituye una buena fuente de tocoferoles.

\section{TABLA 4}

\section{Composición en tocoferoles de aceite de las semillas.}

\section{Tocoferoles (mg/kg)}

$\alpha$-tocoferol

$\beta$-tocoferol

$\gamma$-tocoferol

$\delta$-tocoferol

Tocoferoles totales

Vitamina E (UI/kg)

Vitamina $E^{*}$

\section{Media}

$$
\begin{gathered}
27,2 \pm 0,14 \\
0,2 \pm 0 \\
1,3 \pm 0
\end{gathered}
$$

$\operatorname{tr}$

$$
\begin{gathered}
28,7 \pm 0,13 \\
30 \pm 0 \\
27,38 \pm 0,14
\end{gathered}
$$




\section{CONCLUSIONES}

Las semillas de Eugenia dysenterica DC mostraron ser una importante fuente de carbohidratos totales, pudiendo ser aprovechadas como producto alimenticio destinado al consumo humano.

Las semillas mostraron una relevante actividad antioxidante y un alto contenido de compuestos fenólicos totales.

Entre los ácidos monoinsaturados y poliinsaturados, sólo el ácido oleico y el ácido linoleico se encontraron en cantidades significativas.

En el presente estudio, el aceite de las semillas constituye una baja fuente de tocoferoles comparado con otros aceites y grasas.

\section{RESUMEN}

Este trabajo tuvo por objetivo caracterizar las semillas de Eugenia dysenterica DC en cuanto a su composición proximal, medir su potencial antioxidante, evaluar el perfil de ácidos grasos y determinar la composición de tocoferoles en el aceite extraído de las mismas. Para la obtención del extracto, las semillas deshidratadas y trituradas fueron extraídas con alcohol etílico por 30 minutos, en la proporción de 1:3 de semillas:alcohol etílico, bajo agitación continua, a temperatura ambiente. Seguidamente, la mezcla fue filtrada y el sobrenadante fue deshidratado a $40^{\circ} \mathrm{C}$ con la finalidad de determinar, por pesaje directo, el rendimiento en materia seca del extracto. Las semillas de "cagaita" mostraron ser una importante fuente de carbohidratos totales, además presentaron una relevante actividad antioxidante y un contenido de compuestos fenólicos totales elevado. En su aceite se encontró mayores porcentajes de ácidos grasos palmítico, oleico y linoleico. En comparación con otros aceites y grasas no constituye una buena fuente de tocoferoles.

Palabras clave: semillas, actividad antioxidante, compuestos fenólicos, DPPH ${ }^{\circ}$.

Dirigir la correspondencia a:

Profesora

Neuza Jorge

Departamento de Engenharia e

Tecnologia de Alimentos,

Instituto de Biociências, Letras e Ciências Exatas,

Universidade Estadual Paulista

São José do Rio Preto

Brasil.

CEP: 15054-000

São José do Rio Preto,

Brasil.

Teléfono: 55-17-32212257
Fax : 55-71-32212299

E-mail: njorge@ibilce.unesp.br

Agradecimientos: A la Coordenação de Aperfeiçoamento de Pessoal de Nível Superior - CAPES, por la concesión de beca de Doctorado y al Conselho Nacional de Desenvolvimento Científico e Tecnológico - CNPq, por la beca de Produtividad en Investigación.

\section{BIBLIOGRAFÍA}

1. Silva RSM, Chaves LJ, Naves RV. Caracterização de frutos e árvores de cagaita (Eugenia dysenterica DC.) no sudeste do estado de Goiás, Brasil. Rev Bras Frutic 2001; 23:330-334.

2. Naves RV, Almeida Neto JX, Rocha MR, Borges JD, Carvalho GC, Chaves LJ, Silva VA. Determinação de características físicas em frutos e teor de nutrientes em folhas e no solo, de três espécies frutíferas de ocorrência natural nos cerrados de Goiás. Pesqui Agropecu Trop 1995; 25:107-114.

3. Ribeiro JF, Proença CEB, Almeida SP. Potencial frutífero de algumas espécies frutíferas nativas dos cerrados. Anais da Soc. Brasil. de Frutic. Brasília (Brasil), 1986, pp. 491-500.

4. Corrêa MP. Dicionário de plantas úteis do Brasil, IBDF, Rio de Janeiro, 1984.

5. Brandão M, Ferreira PBD. Flora apícola do cerrado. Informe Agropec 1991; 15, 7-14.

6. Donadio LC, Môro FV, Servidone AA. Frutas brasileiras. Novos Talentos, Jaboticabal, 2002.

7. Almeida SP. Cerrado: aproveitamento alimentar. Embrapa-CPAC, Planaltina, 1998.

8. Franco G. Tabela de composição química dos alimentos. Atheneu, São Paulo, 1992.

9. Amarowicz R, Pegg RB, Rahimi-Moghaddam P, Barl B, Weil JA. Free radical scavenging capacity and antioxidant activity of selected plant species from the Canadian prairies. Food Chem 2004; 84:551-562.

10. White PJ, Xing Y. Antioxidants from cereals and legumes. AOCS Press ed. Natural antioxidants: chemistry, health effects, and applications. Champaign, 1996, p. 25-55.

11. Shahidi F. Natural antioxidants: an overview. AOCS Press ed. Natural antioxidants: chemistry, health effects, and applications. Champaign, 1996, pp. 1-11.

12. Association of Official Agricultural Chemists'. AOCS. Champaign, 1998.

13. Association of Official Methods of Analysis of the AOAC. Maryland, 1995.

14. Brand-Williams W, Cuvelier ME, Berset C. Use of a free radical method to evaluate antioxidant activity. 
Lebensm Wiss Technol 1995; 28:25-30.

15. Singleton VL, Rossi Jr JA. Colorimetry of total phenolics with phosphomolybdic-phosphotungstic acid reagents. Am J Enol Vitic 1965; 16:144-158.

16. Hartman L, Lago RCA. Rapid preparation of fat acids methil esters. Laboratory and Practy 1973; 22:475-476.

17. Fernandes JB, David V, Facchini PH, Silva MF, Filho ER, Vieira PC, Galhiane MS, Pagnocca FC, Bueno OC, Hebling MJ, Victor SR, Santos AMR. Extrações de óleos de sementes de citros e suas atividades sobre a formiga cortadeira Atta sexdens e seu fungo simbionte. Quim Nova 2002; 25:1091-1095.
18. Gaméz-Meza N, Noriega-Rodríguez JA, MedinaJuárez LA, Ortega-García J, Cázarez-Casanova R, Angulo-Guerrero O. Antioxidant activity in soybean oil of extracts from Thompson grape bagasse. JAOCS 1999; 76:1445-1447.

19. El-Adawy TA, Taha KM. Characteristics and composition of different seed oils and flours. Food Chem 2001; 74:47-54.

20. Corsini MS, Jorge N, Miguel AMRO, Vicente E. Perfil de ácidos graxos e avaliação da alteração em óleos de fritura. Quim Nova 2008; 31:956-961.

21. Borges SV, Maia MCA, Gomes RCM, Cavalcanti NB. Chemical composition of umbu (Spondias Tuberosa Arr. Cam) seeds. Quim Nova 2007; 30:49-52. 